\title{
Author Index Vol. 10, 1995
}

Abbas, A. 297 Aboulafia,Y. 17 Amir, J. 60 Ando,M. 41 Andronikou, S. 1 Arbogast, E. 337 Aubry,M.-C. 26 Avni,F. 192 Ayida,G 1.101

Bairaktari, E. 1 Baker, B.W. 119 Bar-El, H. 76 Bar-Nizan, N. 76 Bebbington, M.W. 32 Beckett, G.J. 11 Belfort,M.A. 119 Benifla,J.L. 189 Besse,G.H. 86 Bieniarz, A. 326 Bignon,J.D. 373

Blanc, B. 48 Blot, P. 387 Bollmann, R. 52 Bombard, A.T. 157 Bonneville, F. 373 Boog, G. 373 Boogert,A. 307 Borochowitz, Z. 76 Boubli,L. 48 Brioschi, E. 315 Brivet,F. 204 Brudenell, M.J. 141 Bucourt,M. 106 Burd,L. 333

Cacheux, V. 387 Camelo, J.S., Jr. 322 Campbell, S. 66 Campbell, S.A. 286 Caputo-Mahieu, D. 26 Cardy,D.L.N. 66 Carroll, S.G. 279, 290 Caubel,P. 106 Cesbron, A. 373 Chagnon,C. 48

Chaoui,R. 52

Chasseray, J.E. 106 Chitrit,Y. 106 Christie, A.D. 111 Cohen, E. 192 Coppens, Y. 214 Cravello,L. 48

Dar,H. 76 David, M. 76 Davies,E.T. 290 Degani,S. 200 Delezoide, A.-L. 26 Delfraissy, J.-F. 7

Delneste,D. 192 D'Ercole,C. 48 Diamant,Y.Z. 17 Djaldetti,M. 60 Dommergues, M. 26

Donner,C. 192 Dorfman, S.A. 186 Druart,L. 387 Drugan,A. 37,182 Dumez, Y. 26 Duquette, D. 286 Dvorin,E. 286

Ebrahim, S.A.D. 182 Evans, M.I. 71, 167,182,186, 286

Faria,M. 349

Farine, D. 92

Favre, R. 337

Feinstein, S.J. 408

Fermont, L. 26

Fernandez, H. 204,301

Filidori,M. 106

Fisk, N. 307

Font, G. 333

Frydman,R. 7, 189,204,301

Fujimoto, S. 22

Fukuiya, T. 207

Garbin,O. 337 Gasser,B. 337 Gayffier, A. de 204 Geijn, H.P. van 178

Gershoni-Baruch, R. 37 Goldstein, I. 81 Guihard-Costa, A.M. 215

Harel,L. 60 Harvey, D. 11 Hoffman, E.P. 71,167 Holt,D.E. 11 Holzgreve, W. 147 Homko,CJ. 81 Howie, A.F. 11 Howie, P.W. 111 Hue,M.V. 301 Hume, R.F., Jr. 182 Hurley, R. 11 Hyett,J $\Lambda$. 381

Ikeda,T. 207 Imbert,M.C. 189 Isada,N.B. 182 Ishimaru, T. 134 Israel, N. 76

Jacquetin, B. 86 James, D. 95 Jauniaux, E. 66 Jevon, G. 119 Jewell, W.H. 186 Johnson, A. 131 Johnson, J.-A. 92 Johnson, M.P. 71, 167, 182 Jorge, S.M. 322

Kalache,K. 52 Kaplan, G.P. 83 Kiefer,H. 387 King,M. 71,167 Knisely,A,S. 139 Koch,S. 311 Koyanagi,T. 400 Krivchenia, E.L. 71,286 Kuhlman,Kム. 393 Kustermann, A. 315 
Langston,C. 119 Lapatsanis, P.D. 1 Lapierre, J.-M. 387

415

Larroche, J.C. 215 Lebon,P. 7 Leclaire, M. 48 Lemery, D.J. 326 Lemery,D.R. 86 Leruez,M. 204 Leslie, J. 111 Lewinsky, R.M. 200 Lolis,D. 1 Longmire, S. 119 Lorenzen, J. 147 Louis, L.St. 186 Lowe, E. 111 Lowry,D.L.B. 286 Lysikiewicz, A. 127

McCorquodale, D.J. 333 McCorquodale, M.M. 333 Machan, L. 32 Mac Mahon, R.A. 343 Macones, G.A. 131 Macphail,S. 92 Makinoda, S. 22 Mandelbrot, L. 26 Marchal,P. 204 Markin,L.B. 127 Martinez, F.E. 322 Maslow,A. 408 Matsumoto, T. 134 Mau,H. 52 Meagher, S. 307 Merksamer, R. 76 Metezeau,P. 387 Minifee,P. 119 Mires, G.J. 111 Moise, K.J., Jr 119 Momma, K. 41 Montgomery, L.D. 119 Morinet, F. 204 Morrow, R.J. 92 Moscoso, G. 381 Muller,J.Y. 373 Münnich, Á. 368 Myles,T.D. 333

Nagano, M. 400 Nakano,H. 400 Negishi,H. 22 Nicolaides, K.H. 66, 141,279, 290,297,349,356,381 Nicolini,U. 315 Nicoloso, E. 48

Nieuwint,A. 178 Nitzan,M. 17

Okuyama, K. 22 Oury,J.-F. 387

Palacios,Q. 119 Pandya,P.P. 66 Papaioannou, S. 290 Papp,Z. 368 Paquet,V. 192 Patel,F. 349

Patel,N.B. 111 Paterson, P.J. 343 Pegoraro, E. 71 Petrikovsky, B.M. 83,127 Pfleghaar, K.M. 393

Pokorny,W. 119 Polzin,WJ. 408 Pons,J.-C. 7,189

Quintero, R.A. 71, 167 Qureshi, F. 167

Rabinowitz, R. 17 Rappoport, P. 157 Reece,E.A. 81,311 Regemorter, N. van 192 Reichler,A. 182 Renou,P.M. 343 Ritchie, J.W.K. 92 Robie,D. 119 Robins, R.M. 186 Rodeck,C.H. 101 Rodesch,F. 192 Ryan, G. 92 Rypens,F. 192

Saade,G.R. 119 Sagot,P. 373 Sala, M.M. de 322 Salvesen, D.R. 141 Santolaya-Forgas, J. 86, 326 Sarno,A.P. 408 Satoh,S. 400 Sebire,NJ. 349,356 Serre,A.F. 86 Shekleton, P.A. 343

Sicherman, N. 157 Sivan,E. 311 Skomorovsky, Y. 17 Slomko,Z. 127 Smoleniec, J. 95

Snijders, R.J.M. 349, 356 Soothill, P.W. 101 Spitzer,A.R. 393 Stefos, Th. 1 Straussberg, R. 60

Suita,S. 400 Sujov,P. 37 Suzuki, T. 207 Szabó,M. 368

Tachdjian,G. 387 Tadmor, O. 17 Taguchi,T. 400 Tanaka,M. 207 Tassis, B. 315 Tennstedt, C. 52 Thomas, A. 189 Tilley,D. 131 Tordjman, N. 301 Troalen, F. 204 Tsolas, O. 1

Vadas, A. 37 Vamos,E. 192 Van den Veyver, I. 119 Vasiliadou, A.-D. 1 Veress,L. 368 Ville,Y. 189,204,301 Vincent, Y. 301 Vugt, J.M.G. van 178

Wade,R. 131 Wapner,R.J. 131,393 Warsof,S.L. 326 Weber, P. 337 Weiner,C.P. 173 Wilson, L., Jr. 326 Wilson, R.D. 32 Wittmann, B.K. 32 Wiznitzer, A. 81

Yakubo,K. 207 Yamabe, T. 134 Yamada,H. 22 Yamanouchi, T. 400 Yamashita, H. 134

Yasuhi,I. 134 Yehudai,I. 76

Zoppini, C. 315 Zorn,B. 106 Zuliani, G. 315

416

Author Index Vol. 10,1995 